\title{
Childhood Cerebral Adrenoleukodystrophy: MR Perfusion Measurements and Their Use in Predicting Clinical Outcome after Hematopoietic Stem Cell Transplantation
}

(D)A.M. McKinney, (D). Benson, (DD.R. Nascene, (D). Eisengart, (D) M.B. Salmela, (DD.J. Loes, (D). Zhang, (D). Patel, (D) G.V. Raymond, and (DW.P. Miller

\begin{abstract}
BACKGROUND AND PURPOSE: MR perfusion has shown abnormalities of affected WM in cerebral X-linked adrenoleukodystrophy, but serial data is needed to explore the import of such findings after hematopoietic stem cell transplantation. Our aim was to prospectively measure MR perfusion parameters in patients with cerebral adrenoleukodystrophy pre- and post-hematopoietic stem cell transplantation, and to correlate those measurements with clinical outcome.
\end{abstract}

MATERIALS AND METHODS: Ten patients with cerebral adrenoleukodystrophy prospectively underwent DSC-MR perfusion imaging at $<45$ days pre- (baseline), 30-60 days post-, and 1 year post-hematopoietic stem cell transplantation. MR perfusion measurements in the 10 patients and 8 controls were obtained from the parieto-occipital WM, splenium of the corpus callosum, leading enhancing edge, and normal-appearing frontal white matter. MR imaging severity scores and clinical neurologic function and neurocognitive scores were also obtained. MR perfusion values were analyzed in the patients with cerebral adrenoleukodystrophy at each time point and compared with those in controls. Correlations were calculated between the pre-hematopoietic stem cell transplantation MR perfusion values and 1-year clinical scores, with $P$ value adjustment for multiple comparisons.

RESULTS: At baseline in patients with cerebral adrenoleukodystrophy, both relative CBV and relative CBF within the splenium of the corpus callosum and parieto-occipital WM significantly differed from those in controls $(P=.005-.031)$ and remained so 1 year posthematopoietic stem cell transplantation ( $P=.003-.005)$. Meanwhile, no MR perfusion parameter within the leading enhancing edge differed significantly from that in controls at baseline or at 1 year $(P=.074-999)$ or significantly changed by 1 year post-hematopoietic stem cell transplantation ( $P=.142-.887)$. Baseline Loes scores correlated with 1-year clinical neurologic function $(r=0.813, P<.0001)$, while splenium of the corpus callosum relative CBV also significantly correlated with 1-year neurologic function scale and the neurocognitive full-scale intelligence quotient and performance intelligence quotient scores $(r=-0.730-0.815, P=.007-.038)$.

CONCLUSIONS: Leading enhancing edge measurements likely remain normal post-hematopoietic stem cell transplantation in cerebral adrenoleukodystrophy, suggesting local disease stabilization. Meanwhile, parieto-occipital WM and splenium of the corpus callosum relative $C B V$ and relative $C B F$ values worsened; this change signified irreversible injury. Baseline splenium of the corpus callosum relative CBV may predict clinical outcomes following hematopoietic stem cell transplantation.

ABBREVIATIONS: ALD = adrenoleukodystrophy; $C A L D=$ cerebral X-linked adrenoleukodystrophy; HSCT = hematopoietic stem cell transplantation; K2 = the coefficient obtained by leakage correction of the dynamic bolus; $\mathrm{LEE}=$ leading edge of enhancement; $\mathrm{MRP}=\mathrm{MR}$ perfusion; $\mathrm{NAFWM}=$ normal-appearing frontal white matter; $\mathrm{PH}=$ peak height; $\mathrm{POWM}=$ parietal-occipital white matter; $r$ - = relative; $\mathrm{SCC}=$ splenium of the corpus callosum

A adrenoleukodystrophy (ALD) is a rare, inherited peroxisomal disorder ( 1 in 20,000 live births); about 35\% of males with ALD develop childhood-onset cerebral X-linked adrenoleu-

Received December 6, 2015; accepted after revision February 8, 2016.

From the Departments of Radiology (A.M.M., J.B., D.R.N., M.B.S.) and Pediatrics (J.E., G.R.), Division of Clinical Behavioral Neuroscience, University of Minnesota Masonic Children's Hospital, Minneapolis, Minnesota; Suburban Radiologic Consultants (D.J.L.), Minneapolis, Minnesota; Clinical and Translational Science Institute (L.Z.), University of Minnesota, Minneapolis, Minnesota; Radiology Associates of the Fox Valley (K.P.), Neenah, Wisconsin; and Department of Pediatrics (W.P.M.), Pediatric Blood and Marrow Transplantation Division, University of Minnesota Amplatz Children's Hospital, Minneapolis, Minnesota.

This publication was supported by Grant Number 1UL1RR033183 from the National Center for Research Resources (NCRR) of the National Institutes of Health (NIH) to kodystrophy (cALD). ${ }^{1-5}$ Hematopoietic stem cell transplantation (HSCT) can halt further demyelination and improve survival and neurologic function in cALD. ${ }^{6-10}$ While not fully elucidated, the therapeutic effect of HSCT may relate to relief of the inflamma-

\footnotetext{
the University of Minnesota Clinical and Translational Science Institute (CTSI). Its contents are solely the responsibility of the authors and do not necessarily represent the official views of CTSI or the NIH. University of Minnesota CTSI is part of a national Clinical and Translational Science Award consortium created to accelerate laboratory discoveries into treatments for patients.

Please address correspondence to Alexander M. McKinney, MD, Department of Radiology, Mayo Building, University of Minnesota, 420 Delaware St SE, Minneapolis, MN 55418; e-mail: mckinrad@umn.edu

- Indicates open access to non-subscribers at www.ajnr.org

http://dx.doi.org/10.3174/ajnr.A4773
} 


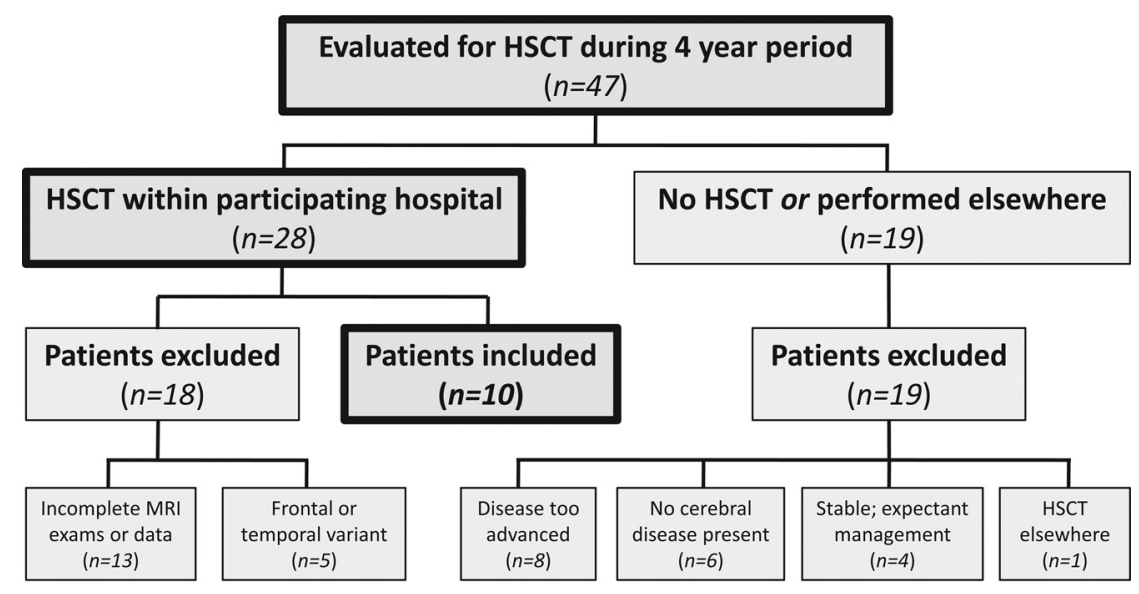

FIG 1. Organizational chart delineating how the 10 patients with cerebral ALD were included for MRP review from the initial 47 patients with ALD.

tion, which is the prominent histologic finding in untreated cALD. ${ }^{5}$ Most important, those with less cerebral disease are more likely to benefit from HSCT; thus, timely HSCT is critical. ${ }^{6-10}$

MR imaging of cALD is vital in predicting disease course and outcomes. The Loes MR imaging severity score, which quantifies the burden of WM disease, correlates with survival and neurologic outcomes after HSCT. ${ }^{6-10}$ The extent of enhancement on postcontrast T1WI, particularly the leading edge of enhancement (LEE), predicts disease progression in untreated cALD, suggesting that blood-brain barrier dysfunction plays a role in CALD. ${ }^{3,11,12}$ Thus, monitoring the LEE and noting that it is halted may be important in defining treatment response.

DSC-MR perfusion parameters such as relative CBV (rCBV), relative $\mathrm{CBF}$ ( $\mathrm{rCBF}$ ), relative TTP ( $\mathrm{rTTP}$ ), relative peak height $(\mathrm{rPH})$, and dynamic leakage correction coefficient (K2) can reflect the pathophysiology of various disorders. ${ }^{12-17}$ Elevated CBV reflects high capillary permeability, while low CBV suggests irreversibly injured tissue. ${ }^{12-17} \mathrm{CBF}$ reflects tissue perfusion, being increased in high-grade tumors or inflammation, while TTP elevation indicates a delay in perfusion. ${ }^{12-17}$ Peak height is the signalintensity change from the baseline of a dynamic enhancement curve, while $\mathrm{K} 2$ is the coefficient obtained by leakage correction of the dynamic bolus, reflecting permeability at sites of blood-brain barrier injury. ${ }^{14,17}$

Scant literature exists regarding MRP parameters in CALD at baseline or how they change following HSCT; 1 sole study of 8 patients noted low rCBV within the core of nonenhancing parietal-occipital white matter (POWM) at baseline, while within the LEE rCBV was preserved. ${ }^{12}$ However, other MRP parameters should be explored, and the prognostic impact of baseline (preHSCT) regional DSC-MRP parameters on outcome is of primary importance. Thus, according to these limited prior data, the hypothesis of this study was that MRP parameters, in particular rCBV, would be abnormal in the POWM and perhaps the splenium of the corpus callosum, compared with these parameters in controls, while perfusion within the LEE would be preserved following successful HSCT. Hence, the aims of this study were the following: 1) to determine whether MRP parameters at baseline in various regions of the cALD-affected brain differ from those of controls, 2) to describe several MRP parameters on the pre- and
post-HSCT cALD MR imaging, and 3) to determine whether any of the measured baseline (pre-HSCT) MRP parameters in various locations correlate with the neurologic outcomes.

\section{MATERIALS AND METHODS Patient Selection}

Institutional review board approval was obtained for this study; all patients were enrolled after informed consent. MR imaging examinations were performed at 3 time points relative to transplantation: baseline pre-HSCT ( $<45$ days before transplantation), 30-60 days postHSCT, and 1 year post-HSCT. There was retrospective measurement of the DSC-MRP values. Patients with cALD were included if they met all of the following criteria: 1) biochemical confirmation of ALD, 2) posterior-type cALD variant (the most common type), 3) clinical and neurocognitive evaluations both at baseline and at 1 year, 4) HSCT being performed between January 2010 and January 2014, 5) younger than 18 years of age and, 6) each MR imaging study adequate for the MRP evaluation (Fig 1). Eight male controls were assessed for DSC-MRP values. Controls lacked any cerebral abnormality on MR imaging (which was typically performed to evaluate the skull base or upper neck), had no previous radiation or chemotherapy, and were matched to the ages of patients with cALD at pre-HSCT (baseline) MR imaging.

\section{MR Imaging Acquisition Technique}

The 3T MR imaging protocol included FLAIR, precontrast 3D T1WI, and postcontrast 3D T1WI. Noncontrast 3D T1WI, necessary for coregistration, used an MPRAGE sequence: TR/TE/NEX/ FOV/parallel factor, $1810 / 3.5 \mathrm{~ms} / 1 / 230 \mathrm{~mm} / 2 ; 0.9 \times 0.9 \times 0.9$ mm isotropic voxel; and 6-minute acquisition. ${ }^{18}$ For postcontrast $3 \mathrm{D}$ T1WI, an intravenous $0.1-\mathrm{mmol} / \mathrm{kg}$ of body weight-based dose of gadopentetate dimeglumine (Magnevist; Bayer HealthCare Pharmaceuticals, Wayne, New Jersey) was administered at $4 \mathrm{~mL} / \mathrm{s}$ for a $10-\mathrm{mL}$ maximum dose (Figs 2-4). The echo-planar DSC-MRP parameters were the following: TR/TE/NEX/FOV/ parallel factor, $1500 / 43 \mathrm{~ms} / 1 / 230 \mathrm{~mm} / 0 ; 1.8 \times 1.8 \times 5 \mathrm{~mm}$ voxel size; and approximately 1 -minute acquisition. The postcontrast 3D T1WI sequence was identical to that of the noncontrast 3D T1WI, being initiated at approximately 10 minutes after the contrast injection.

\section{MRP Postprocessing and Review}

The postprocessing of 38 total DSC-MRP examinations (10 patients with cALD with 3 time points each; 8 controls with 1 study each) was retrospectively processed on a DynaSuite Neuro MR Workstation (Invivo, Gainsville, Florida) by a staff neuroradiologist (A.M.M., with $>10$ years' experience in pediatric neuroimaging) who was blinded to the clinical data. The noncontrast 3D T1WI and FLAIR images were automatically coregistered with the DSC-MRP maps by the postprocessing software and were confirmed visually, with both a neuroradiology fellow and radiology 


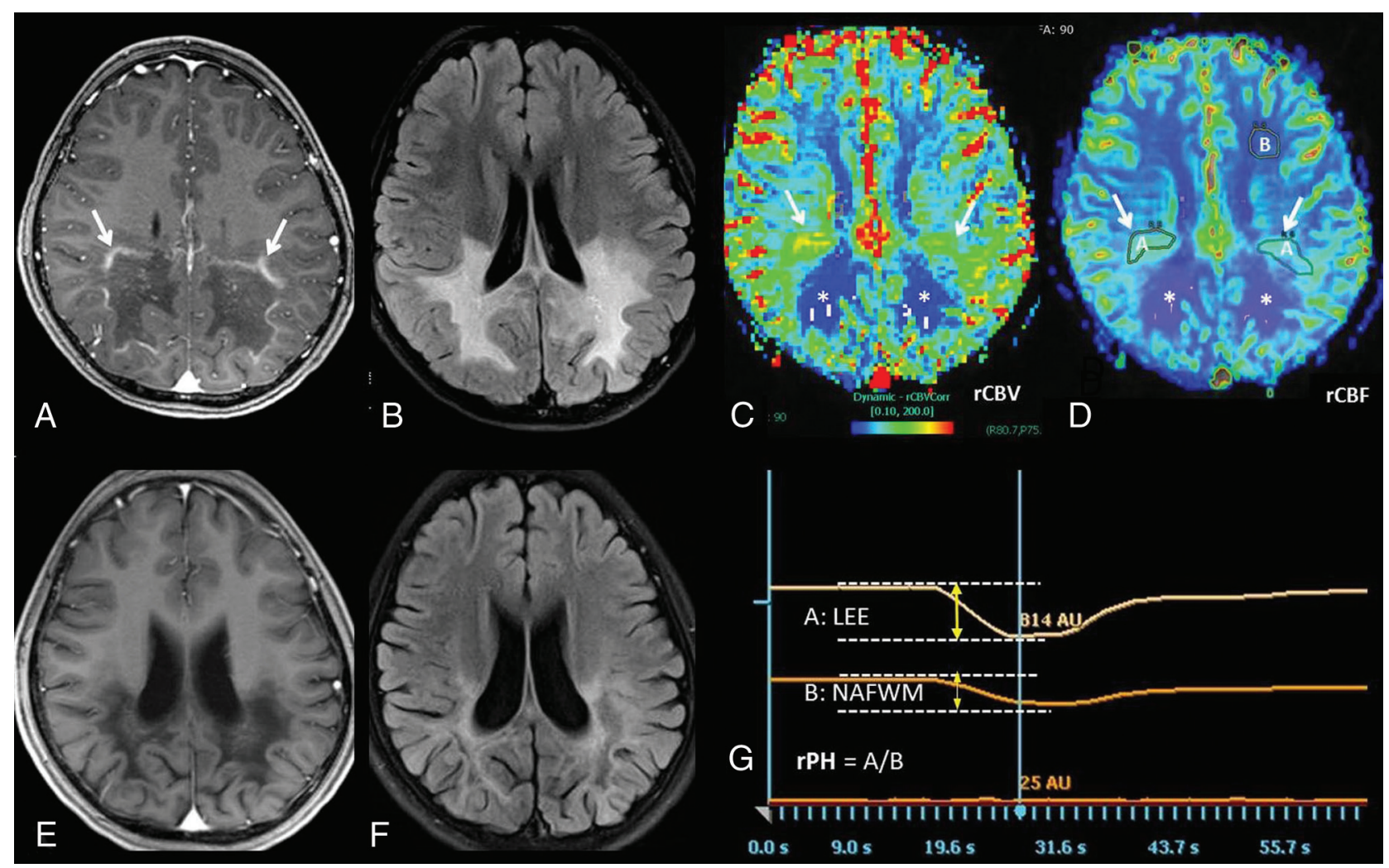

FIG 2. Example of DSC-MRP measurements in a 10-year-old boy with CALD. Baseline (pre-HSCT) MR images show the LEE (arrows) on postcontrast TIWI $(A)$, with typical findings of posterior-type CALD on FLAIR at that time $(B)$. On DSC-MRP at that time, both $\mathrm{rCBV}(C)$ and $\mathrm{rCBF}(D)$ are mildly elevated at the LEE (arrows) but appear low centrally within the POWM (asterisks). G, The method of relative peak height measurement within the LEE and NAFWM ROIs on the baseline (pre-HSCT) MR imaging examination, via measurement of the dynamic contrast curve. Follow-up MR imaging at 1 year shows that the LEE has resolved on postcontrast TTWI $(E)$ and that the WM abnormalities did not progress on FLAIR $(F)$, though moderate overlying parieto-occipital-predominant atrophy has ensued, with interval sulcal enlargement.

resident present to aid in confirming the adequacy of the coregistration, the presence/absence of contrast enhancement, and the ROI site. Freehand ROIs of $\geq 5$-mm diameter were measured centrally within the normal-appearing frontal white matter (NAFWM) and within 3 affected sites: the POWM, centrally within the splenium of the corpus callosum (SCC), and the visibly enhancing LEE (such enhancing regions occurred within areas of FLAIR abnormality in either the POWM or SCC) as demonstrated in Figs 2-4. ${ }^{3,11,12}$ At least 3 ROIs were measured at each site, and the mean was recorded; if lesions were bilateral, the mean of 6 ROIs (3 ROIs on each side) was used. In controls, normal POWM was substituted for the LEE measurement because no enhancement was present. Notably, LEE lesions being either bilateral, unilateral, or midline prevented a side-to-side comparison of MRP values and thus necessitated the incorporation of measurements for both sides for a conglomerate mean of at least 3 MRP parameter values.

After ROI placement, leakage-corrected CBV, CBF, TTP, and K2 maps were automatically generated in patients and controls, while PH was calculated by manual analysis of the dynamic contrast curve (Fig 2). ${ }^{13-18}$ Regarding the LEE, because enhancement typically resolves following HSCT for cALD, the post-HSCT MR images were automatically coregistered to the pre-HSCT MR images, with visual confirmation to ensure that the same anatomic site of the LEE was measured across time. Relative values of each parameter were calculated from the POWM, SCC, or LEE by di- viding by the value of that parameter with the measurement obtained from NAFWM; the exception was that only raw K2 was recorded because relative $\mathrm{K} 2$ cannot be calculated given its zero value within the NAFWM.

\section{MR Imaging Severity Scoring (Loes Score)}

Two neuroradiologists with $>5$ years' experience with cALD (D.J.L, D.R.N.) reviewed the FLAIR images by consensus to generate MR imaging severity (Loes) scores. This review was performed according to the method of prior studies. ${ }^{8,19}$ Both neuroradiologists were blinded to the clinical data.

\section{Measurement of Clinical Outcome}

The gross neurologic function at baseline (pre-HSCT) and 1 year post-HSCT was determined by using a previously described 25point cALD severity neurologic function scale; notably, increasing scores on the scale denotes worsening function. ${ }^{9,10}$ For the cALD cohort, the neurologic function scale was retrospectively constructed from detailed clinical assessments in the medical record by a pediatric HSCT specialist (W.P.M.). ${ }^{9,10,19}$ Neurocognitive scores from the Wechsler Intelligence Scale Series, including full-scale intelligence quotient, performance intelligence quotient, and verbal intelligence quotient, were prospectively obtained by dedicated examination from 1 of several neuropsychologists both pre-HSCT and at 1 year, as described previously. ${ }^{19-21}$ Both the pediatric transplantation specialist 


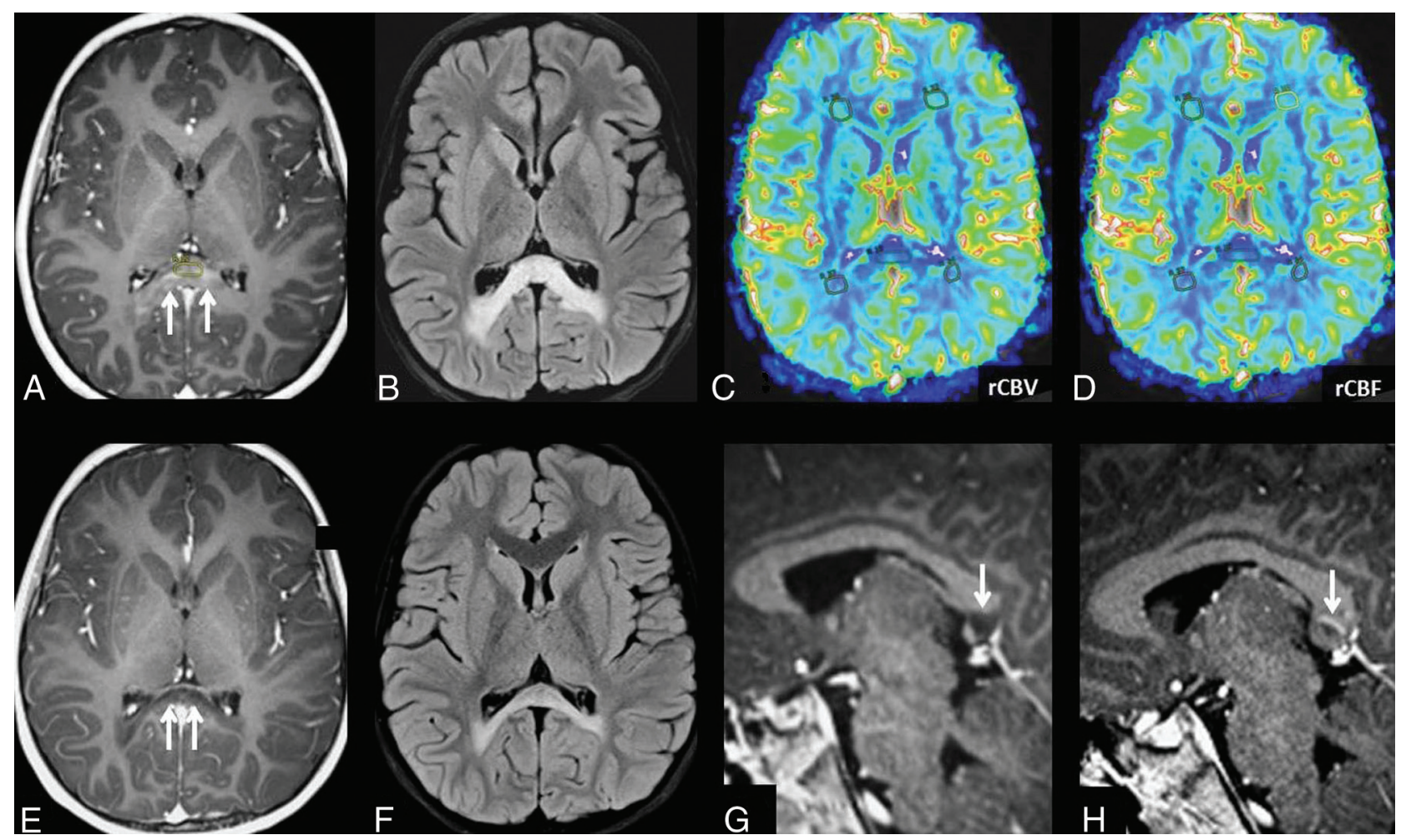

FIG 3. A 6-year-old boy with CALD. Pre-HSCT, a LEE (arrows) is present along the anterior SCC on postcontrast TIWI (A), with edema on FLAIR within the SCC and, to a lesser degree, within the POWM $(B)$. Also at baseline, the $C B V(C)$ and $C B F(D)$ maps demonstrate ROls obtained from the NAFWM, POWM, and SCC. After HSCT, on 1-year follow-up postcontrast TIWI (E), the LEE has disappeared, with decreased swelling and no worsening of the extent on FLAIR (F). Note that the sagittal postcontrast TIWI at 1 year $(G)$ demonstrates the disappearance of the enhancing LEE (arrow) within the SCC, compared with the initial postcontrast image at baseline $(H)$.

and the neuropsychologists were blinded to the MR imaging data.

\section{Statistical Analysis}

Mann-Whitney tests were used to assess differences between patients with cALD and controls regarding the regional DSC-MRP values at each MR imaging time point (notably, the MRP values at a single time point in controls were compared for each of the 3 cALD cohort time points). Intrapatient pre- and post-HSCT MRP values were compared via the linear mixed model, with $P$ value adjustment for multiple comparisons applied by using the Tukey method. Both the pre-HSCT (baseline) MRP values and the Loes scores were correlated with the 1-year clinical outcome functional and neurocognitive scores (neurologic function scale, full-scale intelligence quotient, performance intelligence quotient, and verbal intelligence quotient) by the Spearman method. Statistical analyses were performed by using SAS software (Version 9.3; SAS Institute, Cary, North Carolina). The significance threshold was set to $P<.05$.

\section{RESULTS}

Ten boys with cALD were ultimately included for analysis from the initial population of 47 males with ALD who were evaluated in the clinic during the same period. Reasons for exclusion are provided in Fig 1. Neither the baseline ages nor the Loes scores differed between the included and excluded patients (both $P>.05$ ). The mean age at baseline MR imaging for the 10 patients was $8.2 \pm 2.7$ years (range, $5-14$ years), and for controls, it was $7.8 \pm$
3.2 years (range, $4-12$ years); these ages were not significantly different $(P=.748)$. During the study period, none of the 10 patients with CALD incurred other cerebral pathology that confounded the DSC-MRP evaluation. The mean MRP measurements for the cALD cohort at all 3 time points are provided, with $P$ values when comparing with controls, in Table 1.

Regarding the SCC, both $\mathrm{rCBV}$ and $\mathrm{rCBF}$ were significantly less in patients than in controls at both baseline and 1-year postHSCT (Table 1). At the 30- to 60-day post-HSCT time point, only rPH significantly differed between patients and controls. As for intrapatient $\mathrm{rCBV}$ and $\mathrm{rCBF}$, both parameters decreased from pre- to 1 -year post-HSCT by $5.6 \%$ and $10.0 \%$, respectively; however, such changes were not statistically significant between the 2 time points for either parameter $(P=.057-.076)$.

As for the POWM, there were significant differences between the patients with cALD and controls; rCBV, rCBF, and rTTP differed significantly between patients and controls at all 3 time points (Table 1). Within the cALD cohort, both intrapatient rCBV and rCBF decreased from the pre- (baseline) to 1-year postHSCT time points by $30.7 \%$ and $33.0 \%$, respectively; however, these were not found to be significantly different between the 2 time points for either parameter $(P=.101-.118)$.

All patients had an enhancing LEE before HSCT, and each had resolution of that visibly enhancing edge by 1 year post-HSCT (Figs 2-4). Within the LEE, the day 30-60 rPH was the only MRP parameter that differed significantly between the 10 patients with CALD and controls, while the pre- (baseline) and 1-year post- 


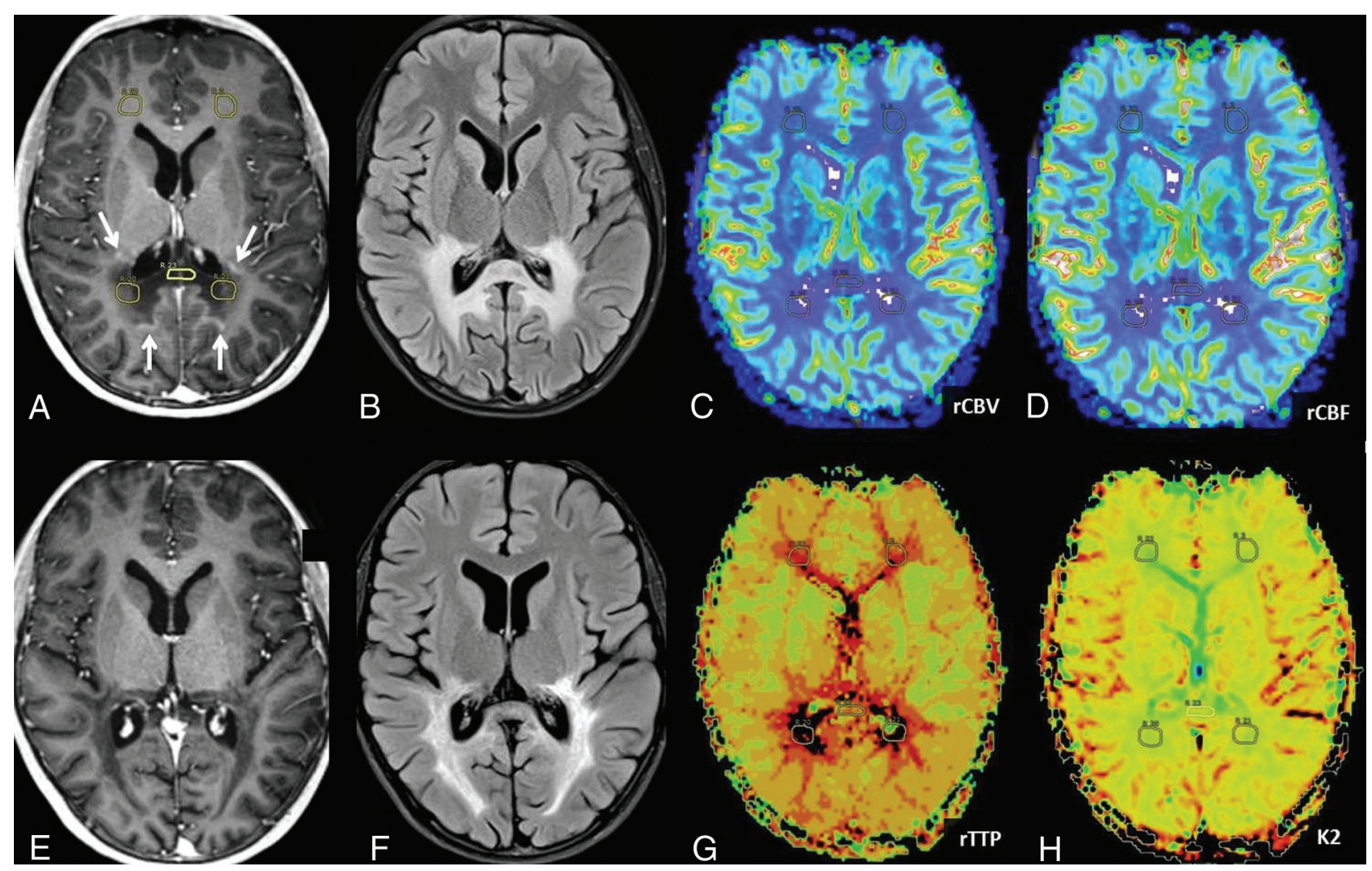

FIG 4. A 15 -year-old adolescent boy with ALD. Pre-HSCT postcontrast TIWI (A) demonstrates a LEE (arrows) in the POWM and optic radiations, with edema on noncontrast FLAIR (B). Baseline pre-HSCT ROIs from the NAFWM, POWM, and SCC are depicted on postcontrast TIWI (A) and on the MRP CBV $(C)$, CBF $(D)$, TTP $(G)$, and K2 $(H)$ maps. After HSCT, the 1-year follow-up MR imaging demonstrates resolution of the LEE on postcontrast TIWI $(E)$, with no overt change in the extent of abnormality on FLAIR (F), though mild-moderate cerebral atrophy has ensued, being more prominent within the SCC.

Table 1: Mean of DSC-MRP parameters with $P$ values comparing patients with ALD with controls at each time ${ }^{a}$

\begin{tabular}{|c|c|c|c|c|c|c|}
\hline \multirow{3}{*}{$\begin{array}{l}\text { DSC-MRP } \\
\text { Parameter }\end{array}$} & \multicolumn{2}{|c|}{ Pre-HSCT } & \multicolumn{2}{|c|}{ Post-HSCT (30-60 Days) } & \multicolumn{2}{|c|}{ Post-HSCT (1 year) } \\
\hline & & $P$ Value versus & & $P$ Value versus & & $P$ Value versus \\
\hline & Mean & Controls & Mean & Controls & Mean & Controls \\
\hline SCC: rCBV & $0.71 \pm 0.32$ & $.005^{b}$ & $1.02 \pm 0.47$ & .404 & $0.67 \pm 0.25$ & $.005^{\mathrm{b}}$ \\
\hline SCC: rCBF & $0.70 \pm 0.34$ & $.005^{\mathrm{b}}$ & $1.00 \pm 0.47$ & .553 & $0.63 \pm 0.24$ & $.003^{b}$ \\
\hline SCC: rTTP & $0.26 \pm 1.83$ & .813 & $0.13 \pm 1.51$ & .887 & $0.35 \pm 2.94$ & .377 \\
\hline SCC: rPH & $0.96 \pm 0.38$ & .093 & $0.85 \pm 0.30$ & $.025^{\mathrm{b}}$ & $0.82 \pm 0.36$ & $.019^{\mathrm{b}}$ \\
\hline SCC: K2 & $0.004 \pm 0.001$ & .177 & $0.00 \pm 0.00$ & .824 & $0.00 \pm 0.00$ & .999 \\
\hline POWM: rCBV & $0.72 \pm 0.30$ & $.031^{\mathrm{b}}$ & $0.63 \pm 0.31$ & $.004^{\mathrm{b}}$ & $0.50 \pm 0.40$ & $.004^{b}$ \\
\hline POWM: rCBF & $0.70 \pm 0.27$ & $.023^{b}$ & $0.61 \pm 0.30$ & $.004^{\mathrm{b}}$ & $0.47 \pm 0.34$ & $.004^{\mathrm{b}}$ \\
\hline POWM: rTTP & $1.92 \pm 1.46$ & $.007^{\mathrm{b}}$ & $1.95 \pm 0.95$ & $<.001^{\mathrm{b}}$ & $0.42 \pm 5.22$ & $.043^{b}$ \\
\hline POWM: rPH & $1.19 \pm 0.26$ & .092 & $0.92 \pm 0.34$ & .552 & $0.87 \pm 0.38$ & .238 \\
\hline POWM: K2 & $0.002 \pm 0.003$ & .662 & $0.00 \pm 0.00$ & .999 & $0.00 \pm 0.00$ & .999 \\
\hline LEE: rCBV & $1.38 \pm 0.63$ & .168 & $1.43 \pm 0.62$ & .301 & $1.25 \pm 0.48$ & .618 \\
\hline LEE: rCBF & $1.39 \pm 0.64$ & .153 & $1.43 \pm 0.66$ & .651 & $1.28 \pm 0.46$ & .554 \\
\hline LEE: rTTP & $0.24 \pm 1.34$ & .982 & $-0.11 \pm 0.88$ & .432 & $-1.29 \pm 3.76$ & .601 \\
\hline LEE: rPH & $1.62 \pm 0.67$ & .074 & $1.43 \pm 0.43$ & $.020^{\mathrm{b}}$ & $1.12 \pm 0.46$ & .459 \\
\hline LEE: K2 & $0.004 \pm 0.005$ & .177 & $0.00 \pm 0.00$ & .662 & $0.003 \pm 0.004$ & .999 \\
\hline
\end{tabular}

${ }^{a}$ The relative MRP ratios in patients with ALD compared with NAFWM.

${ }^{\mathrm{b}} P$ values $<.05$.

HSCT values did not (Table 1). Regarding the LEE within the cALD cohort, the intrapatient rCBV and rCBF both decreased by $9.4 \%$ and $7.9 \%$, respectively, though neither these nor any other measured MRP parameter changed significantly between the baseline and 1-year time points $(P=.142-.887)$.

Regarding the Loes MR imaging severity scores, the mean Loes scores at each time point for the cALD cohort (based on FLAIR) were $6.35 \pm 5.5$ at baseline (pre-HSCT), $6.40 \pm 5.5$ at 30-60 days
post-HSCT, and $7.90 \pm 5.6$ on the 1-year follow-up MR imaging examinations. As would be expected on the basis of prior literature, the pre-HSCT Loes score strongly correlated with the 1-year clinical neurologic function scale score $(r=0.813, P<$ $.0001) .^{9,10,19}$ Regarding the neurocognitive measures, moderate correlations existed between the baseline Loes score and the 1-year neurocognitive full-scale intelligence quotient, performance intelligence quotient, and verbal intelligence quotient 
Table 2: Correlations between both the pre-HSCT MRI severity and MRP parameters with the 1-year follow-up clinical outcome scores ${ }^{a}$

\begin{tabular}{lcccc}
\hline \multicolumn{1}{c}{ Parameter } & NFS & FSIQ & PIQ & \multicolumn{1}{c}{ VIQ } \\
\hline SCC: rCBV & $-0.730, .016^{\mathrm{b}}$ & $0.735, .038^{\mathrm{b}}$ & $0.815, .007^{\mathrm{b}}$ & $0.500, .171$ \\
SCC: rCBF & $-0.451, .191$ & $0.590, .123$ & $0.678, .045^{\mathrm{b}}$ & $0.233, .546$ \\
SCC: $r T T P$ & $-0.291, .414$ & $0.205, .627$ & $0.033, .932$ & $0.400, .286$ \\
SCC: $r$ PH & $0.118, .745$ & $-0.012, .977$ & $0.059, .881$ & $-0.017, .966$ \\
POWM: rCBV & $-0.555, .096$ & $0.325, .432$ & $0.301, .431$ & $0.367, .332$ \\
POWM: rCBF & $-0.557, .095$ & $0.325, .432$ & $0.301, .431$ & $0.360, .342$ \\
POWM: rTTP & $0.569, .086$ & $-0.663, .073$ & $-0.619, .075$ & $-0.550, .125$ \\
POWM: rPH & $-0.326, .358$ & $-0.036, .932$ & $-0.126, .748$ & $0.250, .517$ \\
LEE: rCBV & $0.045, .901$ & $-0.217, .606$ & $-0.126, .748$ & $-0.326, .391$ \\
LEE: rCBF & $-0.090, .804$ & $0.024, .955$ & $0.042, .915$ & $-0.017, .967$ \\
LEE: rTTP & $0.083, .819$ & $-0.217, .606$ & $-0.226, .559$ & $-0.150, .700$ \\
LEE: rPH & $0.111, .760$ & $-0.506, .201$ & $-0.452, .222$ & $-0.250, .517$ \\
Loes sCore & $0.813,<.0001^{\mathrm{b}}$ & $-0.646, .084$ & $-0.633, .068$ & $-0.596, .090$ \\
\hline
\end{tabular}

Note:-NFS indicates neurologic function scale; FSIQ, full-scale intelligence quotient; PIQ, performance intelligence quotient; VIQ, verbal intelligence quotient.

a Numbers are rounded to the one-thousandth place. In addition, the correlations for $\mathrm{K} 2$ are not shown because most measurements were 0.00

${ }^{\mathrm{b}} \mathrm{P}$ values $<.05$

scores, but these did not reach clinical significance $(r=-0.596$ to $-0.646, P=.068-.090)$ after correction for multiple comparisons (Table 2).

Regarding correlating the baseline DSC-MRP parameters with the functional and neurocognitive scores at 1 year post-HSCT, the only parameters that correlated with the clinical and neurocognitive outcomes were rCBV and $\mathrm{rCBF}$ within the SCC (Table 2). In particular, baseline SCC rCBV had an inverse, significant correlation with the clinical neurologic function scale score at 1 year $(r=-0.730, P=.016)$ and a positive correlation with the neurocognitive scores full-scale intelligence quotient $(r=0.735, P=$ $.038)$ and performance intelligence quotient $(r=0.815, P=$ $.007)$; there was a positive, significant correlation also found between baseline SCC rCBF and performance intelligence quotient ( $r=0.678, P=.045)$. Notably, no significant correlation was found between any regional MRP parameter and the 1-year neurocognitive verbal intelligence quotient scores, after correction for multiple comparisons. Additionally, no MRP measure within the POWM or LEE significantly correlated with the 1-year neurologic function scale or neurocognitive outcome scores (each $P>.05$ ).

\section{DISCUSSION}

Because childhood cALD is typically a progressive and severe disorder in the absence of HSCT, the primary goals of this study were the following: 1) to determine whether MRP parameters differ from those in controls in various regions of the cALD-affected brain, 2) to describe a variety of DSC-MRP parameters both preand post-HSCT, and 3) to determine whether any baseline (preHSCT) MRP markers portend the clinical outcome following HSCT. ${ }^{6-10}$ Regarding the former goal, both this study and that of Musolino et $\mathrm{al}^{12}$ seem to be in accord, because this study confirms that in untreated CALD, rCBV within the "core" of nonenhancing POWM but not the LEE is significantly lower than that in controls. In our transplanted cohort, this difference in rCBV from controls persisted at 1 year following HSCT. Such findings indicate that the core of abnormal POWM (termed "Zone A" in the prior study by Musolino et $\mathrm{al}^{12}$ ) likely comprises severely injured and perhaps nonviable tissue. Accordingly, slowly progressing parieto-occipital regional atrophy typically develops in this region, which was also observed in the current study. ${ }^{12}$ Meanwhile, the arrest of enhancement at the LEE, along with the preserved rCBV and $\mathrm{rCBF}$ at 1 year postHSCT, would imply viable tissue therein. Notably, in our cohort, the POWM rCBV was, on average, about $50 \%$ of that of the NAFWM, whereas the rCBV was about $20 \%$ of that of the NAFWM in the prior study. ${ }^{12}$ This difference in rCBV could relate to the higher mean Loes score (13.4 versus 6.4 in this study) in the prior study, perhaps reflecting more severe tissue injury overall. $^{12}$

The mechanism of how HSCT attenuates the neuroinflammation in CALD is unknown; however, the normal rCBV within the LEE that persists after HSCT suggests a correction of the blood-brain barrier abnormality, along with preservation of regional parenchyma., ${ }^{3,5,10-12}$ Similarly, the study by Musolino et $\mathrm{al}^{12}$ found that $\mathrm{rCBV}$ was preserved in the LEE (termed "Zone B") but was lower in adjacent, centrifugally located areas (Zone C); hence, the current study corroborates the Zone B findings of that study. Such an arrest in demyelination may have a histopathologic precedent, in which murine studies have shown that certain microglial cells are absent initially at the demyelinating edge, but there is a subsequent slow return of microglial guard cells by about 1 year post-HSCT as progression of the disease halts. ${ }^{22-26}$ Thus, 1 theory, which remains speculative, is that blood-brain barrier dysfunction is actually helpful or is required for the marrow-derived precursor cells to return into the cerebrum and differentiate into microglia because a LEE is nearly always present before HSCT in those patients who have undergone a successful HSCT and ultimately have stabilized cerebral disease. ${ }^{22-26}$ Thus, this theory suggests that microglia reflect an overall return of hematopoietic precursor stem cells to the cerebrum, resulting in the observed disease arrest, along with the subsequent repair. ${ }^{22-26}$ Zonal measurements within and adjacent to the LEE were not the focus of the current study but could be considered in future ones.

Regarding the SCC, this aforementioned theory may, at least in part, explain the lack of a significant difference in the large majority of the various MRP measures at 30-60 days post-HSCT, even though there were significant differences at baseline and at 1 year post-HSCT (especially in $\mathrm{rCBV}$ and $\mathrm{rCBF}$ ). ${ }^{22-26}$ Thus, we surmise that there could be both components of irreversible injury and healing of the blood-brain barrier occurring macroscopically in the SCC, which may be an "inflection point" in the disease process. For example, according to the above-mentioned theory, HSCT can correct the underlying microglial dysfunction by way of bone marrow resident and progenitor cells entering the cerebrum through a patent and abnormal blood-brain barrier via capillary recruitment and resultant increased microperfusion; we opine that this might be represented by a transient relatively decreased rTTP and relatively increased $\mathrm{rCBV}$ and $\mathrm{CBF}^{22-26}$ In the current study, such transient changes (ie, increased $\mathrm{rCBF}$ and $\mathrm{rCBV}$ ) were indeed noted in the SCC, where the rTTP transiently 
decreased at 30-60 days post-HSCT and then rose again to above baseline at 1 year post-HSCT. However, by 1 year post-HSCT, both SCC rCBF and rCBV decreased to below baseline, suggesting that such increased perfusion at 30-60 days post-HSCT was indeed only a temporary phenomenon.

Hence, the measurements at 30-60 days post-HSCT might reflect an attempt for the cerebrum to undergo healing by temporarily increasing perfusion to the SCC by a vascular response, though there is also likely a component of underlying irreversible injury, as evidenced by the lower 1-year $\mathrm{rCBV}$ and $\mathrm{rCBF}$ values. If so, then this combination of findings might explain the SCC being the 1 site where the baseline DSC-MRP measurements predict the neurocognitive outcomes (hence, the term "inflection point") because it is a region that has both viable tissue that can potentially be saved, along with nonviable tissue. In contrast, the POWM appears to be a region where the tissue injury is largely irreversible, as evidenced by the lack of such transient findings at 30-60 days post-HSCT. Accordingly, within the POWM, rTTP remained higher and $\mathrm{rCBV}$ and $\mathrm{rCBF}$ both progressively decreased after HSCT. Thus, the POWM seems mostly unaffected by successful transplantation.

This study also found that the baseline SCC rCBV and rCBF values strongly correlated with various measures of neurologic function at 1 year after successful HSCT, perhaps due to the reasons described above. While the Loes MR imaging score is known to correlate with the gross neurologic outcome after HSCT, the findings in the current study suggest that regional DSC-MRP values could augment the Loes score in predicting outcomes better. ${ }^{7-11}$ However, this study was limited, in that most boys within the cohort had generally favorable baseline Loes scores. Hence, these findings may not be applicable to those patients with more severe initial disease. Thus, the utility of MRP in predicting outcomes in patients with cALD with more severe disease needs to be further studied.

There has been an ongoing search for the best baseline (preHSCT) imaging marker of clinical outcome following transplantation (HSCT). Regarding such biomarkers, to our knowledge, the Loes score is the most reproducible and strongest predictor of outcome, being proved across multiple centers. ${ }^{6-10}$ While the Loes score is a "continuous" marker of radiographic severity between 0.5 (minimum nonzero score) and 34 (maximum score), its predictive value mostly rests on a binary correlation with quite broad outcomes. Specifically, boys with a score of $<10$ generally do well after HSCT, while those with a Loes $\geq 10$ generally do poorly after transplantation. Thus, there is a need for imaging biomarkers within both subgroups of less affected (ie, Loes of $<10$ ) and more severely affected (ie, Loes of $\geq 10$ ) patients at baseline. Thus, to our knowledge, there has been no baseline imaging biomarker within the less affected subgroup shown to correlate with eventual posttransplantation neurocognition; the current data suggest that DSC-MRP could be of some utility in this subgroup. Meanwhile, within a subgroup of patients with higher Loes scores $(\geq 10)$, a recent study found that the intensity of visible enhancement on postcontrast T1WI may lend predictive value to clinical outcome, and this was found to be relatively simple to measure, as well as reproducible between observers. ${ }^{27}$

Limitations of this study include the relatively small size of the patient cohort with cALD. Thus, the expense of travel, care, and MR imaging for multiple trips is a known factor contributing to this limitation. ${ }^{19}$ Similarly, it is difficult to obtain a large number of age-matched controls for MRP because most pediatric indications for MR imaging do not necessitate contrast. A third potential limitation was the use of relative rather than absolute perfusion measurements; however, relative measurements of CBV or $\mathrm{CBF}$ are typically more reproducible than absolute ones. ${ }^{15,16} \mathrm{Ad}-$ ditionally, a limitation could be the lack of interobserver reliability calculations between readers regarding the $3+$ ROI placements $(6+$ if the measurement was bilateral) and subsequent MRP values obtained. However, it was not feasible to have 2 readers, given the expertise needed in interpretation of CALD along with familiarity with the postprocessing software for coregistration and MRP calculation; such postprocessing steps including the MRP measurements and calculations were too exhaustive and time-consuming for multiple readers. Particularly, the numerous steps in this process could inherently cause variability among observers.

Finally, a limitation may be that the patient cohort of this study demonstrated relatively low pretransplantation Loes scores (eg, compared with a study by Musolino et $\mathrm{al}^{12}$ ), while there was no significant difference in scores between the excluded $(n=37)$ and included cohort $(n=10)$; this could raise the question of an underlying selection bias in our patient pool. ${ }^{6-12}$ Thus, factors that decreased the mean Loes score in the excluded group were that 6 patients had no cerebral disease (Loes score of 0 ), while 4 were stable (having very low Loes scores near zero); however, this apparent lowering in mean Loes score at baseline may, to some degree, be counterbalanced because some of the excluded patients had disease too severe to be transplanted $(n=8)$ or they did not travel back after transplantation for each MR imaging due to severe dysfunction $(n=13)$ (Fig 1$)$.

Meanwhile, among the 10 included patients, the mean Loes score was relatively low because those with very high scores are very unlikely to undergo transplantation, for example, compared with the prior study by Musolino et $\mathrm{al},{ }^{12}$ which focused on patients with higher mean Loes scores. Hence, there may be an inherent selection bias in this study because those with much higher Loes scores are not likely to undergo HSCT and thus would be excluded, while those patients who did undergo HSCT but were too sick or dysfunctional to travel and return for an adequate follow-up MR imaging were also likely to be excluded. Therefore, as mentioned previously, the findings of this study regarding the clinical response to HSCT cannot be generalized to the subset of patients with cALD who present with much higher Loes MR imaging severity scores on initial evaluation for HSCT. The prediction of outcomes, based on MRI, may be better addressed by gadolinium-intensity measurements, which has been a focus of a recent study by Miller et al. ${ }^{27}$

\section{CONCLUSIONS}

This study found that certain DSC-MRP values in patients with CALD, as measured within the POWM and SCC pre-HSCT, are significantly different from those in controls, while the LEE values are not. With time, rCBV within the LEE remained normal in the patients with cALD relative to controls. This outcome suggests stabilization of a dysfunctional blood-brain barrier at the LEE; conversely, the finding that $\mathrm{rCBV}$ worsens within the POWM following HSCT suggests irreversible injury. This study also found that the $\mathrm{rCBV}$ is the only baseline MRP measurement that 
seems to be a reliable predictor of clinical outcome. Thus, MRP could be a useful adjunct to the Loes MR imaging severity score in predicting the functional and neurocognitive outcomes of boys with cALD with lower Loes scores who are to undergo HSCT.

Disclosures: Alexander M. McKinney—UNRELATED: Board Membership: Vital Images, a division of Toshiba Medical, Minneapolis, Minnesota, Comments: less than $\$ 5000$ reimbursement for travel and consultation per year; Expert Testimony: legal consultation, less than $\$ 10,000$ total this year. Daniel J. Loes-UNRELATED: Consultancy: Bluebird Bio, Comments: consultant for Bluebird Bio research Phase 2 and Phase 3 trials involving viral vector gene therapy for adrenoleukodystrophy. Gerald Raymond-UNRELATED: Consultancy: Minoryx (Barcelona, Spain), Comments: consultant for a company developing products to treat adult adrenoleukodystrophy; Expert Testimony: Department of Health and Human Services, Comments: served as expert in the Division of Vaccine Injury; Grants/Grants Pending: Bluebird Bio (Cambridge, Massachusetts), ${ }^{*}$ Comments: participating in industry-funded research in ALD gene therapy. *Money paid to the institution.

\section{REFERENCES}

1. Moser HW, Smith KD, Watkins PA, et al. X-linked adrenoleukodystrophy. In: Scriver CR, Beaudet AL, Sly WS, et al, eds. The Metabolic and Molecular Bases of Inherited Disease. 8th ed. New York: McGrawHill; 2001:3257-301

2. Bezman L, Moser HW. Incidence of X-linked adrenoleukodystrophy and the relative frequency of its phenotypes. Am J Med Genet 1998;76:415-19 Medline

3. Melhem ER, Loes DJ, Georgiades CS, et al. X-linked adrenoleukodystrophy: the role of contrast-enhanced MR imaging in predicting disease progression. AJNR Am J Neuroradiol 2000;21:839-44 Medline

4. Schaumburg HH, Powers JM, Raine CS, et al. Adrenoleukodystrophy: a clinical and pathological study of 17 cases. Arch Neurol 1975;33:577-91 Medline

5. Powers JM, Pei Z, Heinzer AK, et al. Adreno-leukodystrophy: oxidative stress of mice and men. J Neuropathol Exp Neurol 2005;64: 1067-79 CrossRef Medline

6. Shapiro E, Krivit W, Lockman L, et al. Long-term effect of bonemarrow transplantation for childhood onset cerebral X-linked adrenoleukodystrophy. Lancet 2000;356:713-18 CrossRef Medline

7. Peters C, Charnas LR, Tan Y, et al. Cerebral X-linked adrenoleukodystrophy: the international hematopoietic cell transplantation experience from 1982 to 1999. Blood 2004;104: 881-88 CrossRef Medline

8. Loes DJ, Hite S, Moser H, et al. Adrenoleukodystrophy: a scoring method for brain MR observations. AJNR Am J Neuroradiol 1994;15: 1761-66 Medline

9. Miller WP, Rothman SM, Nascene D, et al. Outcomes following allogeneic hematopoietic cell transplantation for childhood cerebral adrenoleukodystrophy: the largest single-institution cohort report. Blood 2011;118:1971-78 CrossRef Medline

10. Moser H, Raymond G, Koehler W, et al. Evaluation of the preventive effect of glyceryl trioleate-trierucate ("Lorenzo's oil") therapy in $\mathrm{X}$-linked adrenoleukodystrophy: results of two concurrent trials. Adv Exp Med Biol 2003;544:369-87 CrossRef Medline

11. Loes DJ, Fatemi A, Melhem ER, et al. Analysis of MRI patterns aids prediction of progression in X-linked adrenoleukodystrophy. $\mathrm{Neu}$ rology 2003;61:369-74 CrossRef Medline
12. Musolino PL, Rapalino O, Caruso P, et al. Hypoperfusion predicts lesion progression in cerebral X-linked adrenoleukodystrophy. Brain 2012;135:2676-83 CrossRef Medline

13. Grandin CB, Duprez TP, Smith AM, et al. Which MR-derived perfusion parameters are the best predictors of infarct growth in hyperacute stroke? Comparative study between relative and quantitative measurements. Radiology 2002;223:361-70 CrossRef Medline

14. Cha S, Lupo JM, Chen MH, et al. Differentiation of glioblastoma multiforme and single brain metastasis by peak height and percentage of signal intensity recovery derived from dynamic susceptibilityweighted contrast-enhanced perfusion MR imaging. AJNR Am J Neuroradiol 2007;28:1078-84 CrossRef Medline

15. Knopp EA, Cha S, Johnson G, et al. Glial neoplasms: dynamic contrast-enhanced T2*-weighted MR imaging. Radiology 1999;211: 791-98 CrossRef Medline

16. Wetzel SG, Cha S, Johnson G, et al. Relative cerebral blood volume measurements in intracranial mass lesions: interobserver and intraobserver reproducibility study. Radiology 2002;224:797-803 CrossRef Medline

17. Brubaker LM, Smith JK, Lee YZ, et al. Hemodynamic and permeability changes in posterior reversible encephalopathy syndrome measured by dynamic susceptibility perfusion-weighted MR imaging. AJNR Am J Neuroradiol 2005;26:825-30 Medline

18. Boxerman JL, Schmainda KM, Weisskoff RM. Relative cerebral blood volume maps corrected for contrast agent extravasation significantly correlate with glioma tumor grade, whereas uncorrected maps do not. AJNR Am J Neuroradiol 2006;27:859-67 Medline

19. McKinney AM, Nascene D, Miller WP, et al. Childhood cerebral $\mathrm{X}$-linked adrenoleukodystrophy: diffusion tensor imaging measurements for prediction of clinical outcome after hematopoietic stem cell transplantation. AJNR Am J Neuroradiol 2013;34:641-49 CrossRef Medline

20. Wechsler D. Wechsler Intelligence Scale for Children-Fourth Edition (WISC-IV). San Antonio: Psychological Corporation; 2003

21. Riva D, Bova SM, Bruzzone MG. Neuropsychological testing may predict early progression of asymptomatic adrenoleukodystrophy. Neurology 2000;54:1651-55 CrossRef Medline

22. Cartier N, Aubourg P. Hematopoietic stem cell transplantation and hematopoietic stem cell gene therapy in X-linked adrenoleukodystrophy. Brain Pathol 2010;20:857-62 CrossRef Medline

23. Eichler FS, Ren JQ, Cossoy M, et al. Is microglial apoptosis an early pathogenic change in cerebral $\mathrm{X}$-linked adrenoleukodystrophy? Ann Neurol 2008;63:729-42 CrossRef Medline

24. Cartier N, Hacein-Bey-Abina S, Bartholomae CC, et al. Hematopoietic stem cell gene therapy with a lentiviral vector in $\mathrm{X}$-linked adrenoleukodystrophy. Science 2009;326:818-23 CrossRef Medline

25. Kennedy DW, Abkowitz JL. Kinetics of central nervous system microglial and macrophage engraftment: analysis using a transgenic bone marrow transplantation model. Blood 1997;9:986-93 Medline

26. Mildner A, Mack M, Schmidt H, et al. CCR2 + Ly-6Chi monocytes are crucial for the effector phase of autoimmunity in the central nervous system. Brain 2009;132:2487-500 CrossRef Medline

27. Miller WP, Mantovani LF, Muzic J, et al. Intensity of MRI gadolinium enhancement in cerebral adrenoleukodystrophy: a biomarker for inflammation and predictor of outcome following transplantation in higher risk patients. AJNR Am J Neuroradiol 2016;37:367-72 CrossRef Medline 